An Evaluation of Parent-Child Interaction Therapy with and without Motivational

\title{
Enhancement to Reduce Attrition
}

\author{
Haley J. Webb ${ }^{1}$ \\ Rae Thomas ${ }^{2}$ \\ Leanne McGregor ${ }^{1}$ \\ Elbina Avdagic ${ }^{1}$
}

Melanie J. Zimmer-Gembeck ${ }^{1}$

\footnotetext{
${ }^{1}$ School of Applied Psychology and Menzies Health Institute of Queensland, Griffith University, Gold Coast, Australia 4222.

${ }^{2}$ Centre for Research in Evidence-Based Practice, Faculty of Health Sciences and Medicine, Bond University, Gold Coast, Australia, 4226.
}

Corresponding Author:

Haley Webb

School of Applied Psychology

Behavioural Basis of Health,

Griffith University,

Gold Coast Australia. 4222

Email: haley.webb@griffithuni.edu.au

Phone: 56788688 
Acknowledgements: This study was partially supported by funding from Future Directions Prevention and Early Intervention Trials, Queensland Department of Communities, Child Safety and Disability Services, Australia to the Family Interaction Program. The authors wish to thank all members of the Family Interaction Program for helping hundreds of families and collecting data along the way. Our biggest thanks go to the families who have shared their experiences with us and enriched our lives. 


\begin{abstract}
Objective: Although many interventions for child externalizing behavior report promising outcomes for families, high attrition prior to program completion remains a problem. Many programs report dropout rates of $50 \%$ or higher. In this trial we sought to reduce attrition and improve outcomes by augmenting a well-known evidence-based intervention, Parent-Child Interaction Therapy (PCIT), with a 3-session individual motivational enhancement component. Method: Participants were 192 Australian caregivers (91.7\% females; $M_{\text {age }}=34.4$ years) and their children $\left(33.3 \%\right.$ females; $M_{\text {age }}=4.4$ years). Families (51\% referred from child welfare or health services for risk of maltreatment) were assigned to PCIT or a supported waitlist, with families assigned to PCIT receiving either standard PCIT (S/PCIT) or motivation enhanced PCIT (M/PCIT), depending on their time of entry to the study. Waitlist families received phone calls every week for 12 weeks. Results: Parents in M/PCIT reported more readiness to change their behavior from pre-assessment to after the motivation sessions. Also, parents who reported high, rather than low, motivation at pre-assessment did have a lower attrition rate, and there was some evidence that enhancing motivation was protective of premature attrition to the extent that caregivers achieved a high degree of change in motivation. Yet, comparison of attrition rates and survival analyses revealed no difference between M/PCIT and S/PCIT in retention rate. Finally, there were greater reductions in externalizing and internalizing child behavior problems and parental stress among families in S/PCIT and M/PCIT compared with waitlist, and there was generally no significant difference between the two treatment conditions.
\end{abstract}

Keywords: Parent-Child Interaction Therapy; PCIT; Motivational enhancement; attrition; evidence-based treatment 


\section{An Evaluation of Parent-Child Interaction Therapy with and without Motivational Enhancement to Reduce Attrition}

Externalizing child behavior, including aggressive behavior and excessive tantrums, is the most common reason parents seek professional help for themselves and their children (Dishion \& Patterson, 2006; Hiscock et al., 2011). These behavior problems are often chronic and have significant implications for the health and well-being of individuals, families, and communities. For example, adults who experienced childhood psychological problems compared with physical problems had significantly reduced family incomes at age 50 years (Goodman, Joyce, \& Smith, 2011). To address behavior problems early in life, there are now numerous evidence-based parent management training programs with robust findings of effectiveness (e.g., the Incredible Years, Triple P Positive Parenting Program, Parent-Child Interaction Therapy, and Oregon Model Parent Management Training; de Graaf, Speetjens, Smit, de Wolff, \& Tavecchio, 2008; Odgen \& Hagen, 2008; Sanders, Kirby, Tellegen, \& Day, 2014; Thomas \& Zimmer-Gembeck, 2007; Webster-Stratton \& Reid, 2010).

However, across all these programs, retaining families in treatment in order to produce positive outcomes remains a key challenge, with many studies reporting attrition rates of 50\% or higher. A recent meta-analysis of attrition in child and adolescent mental health interventions reported rates ranging from 16 to $72 \%$ depending on attrition definition and study design (de Haan, Boon, de Jong, Hoeve, \& Vermeiren, 2013). Even when attrition was conservatively defined as whether the therapist perceived a family had met treatment goals (agreed, meaning the cessation of treatment appropriate; disagreed, meaning attrition from program), the mean attrition rate was $26 \%$ in efficacy studies and $45 \%$ in effectiveness studies. When attrition was defined as cessation of treatment before a predefined number of treatment sessions, the mean attrition rate from efficacy studies was $29 \%$, compared with $60 \%$ in effectiveness studies. Importantly, two of the strongest predictors of attrition were parents' perception of barriers, where reporting a lower level of perceived barriers was a 
protective factor $(g=0.85)$, and parents' perceptions of the relevance of the treatment, where lower relevance predicted attrition ( $g=0.81$; de Haan et al., 2013). Thus, one of the primary challenges for effective evidence-based parenting interventions is the initial and continuing engagement of parents in the intervention process by reducing perceived barriers and increasing perceived treatment relevance.

\section{Parent-Child Interaction Therapy}

Parent-Child Interaction Therapy (PCIT; Eyberg \& Robinson, 1982) is an individual parent management training intervention for young children (usually children age 3 to 6 years) with externalizing behaviors and their parents. The vast majority of PCIT sessions are direct coaching to increase parent's sensitivity, positive interactions, and adaptive behavior management strategies with his/her child via an ear-piece while the therapist observes parentchild interactions through a one-way mirror (McNeil \& Hembree-Kigin, 2010). In the original time-variable PCIT protocol, treatment progression and conclusion was determined on the basis of parental achievement of mastery criteria, however, more recently, PCIT limited to 12 coaching sessions (referred to in this paper as standard 12-week PCIT), has been found to produce comparable outcomes (Thomas \& Zimmer-Gembeck, 2012). Given these findings and the reduced costs to both caregivers and providers of standard 12-week PCIT, this format was used in the present study.

Whereas engaging in a parenting program is beneficial for many parents of children with challenging behaviors, the level of engagement and motivation required for continued participation can vary across programs depending on whether the parent is an active participant or a passive receiver of information. Perceptions of therapy relevance and motivation to change one's own parenting behaviors are considered particularly important prerequisites for interventions based on active participation, given the higher levels of engagement and motivation required from parents (Nock \& Ferriter, 2005). PCIT is an 
effortful intervention that requires active participation from parents and children. Hence, perceptions of treatment relevance and subsequent motivation were expected to be particularly important for maintaining more families through to treatment completion. Notably, perusal of the PCIT literature reveals that attrition rates typically range from around $25 \%$ to $69 \%$ (Nixon, Sweeney, Erickson, \& Touyz, 2003; Lanier et al., 2011), with various indicators of social disadvantage arising as predictors of attrition, including low maternal age, education, intelligence or family income, and higher maternal psychopathology (Bagner \& Graziano, 2013; Fernandez \& Eyberg, 2009). Moreover, while not specifically examined for PCIT, and rarely studied in relation to parent training in general, attrition or disengagement from parent training programs is sometimes found to be lower among Caucasian parents when compared to other parent groups (e.g., Holden, Lavigne, \& Cameron, 1990; Nix, Bierman, \& McMahon, 2009).

When the results described are considered together with the meta-analytic findings that the strongest predictors of attrition from child and adolescent mental health interventions are parent's perception of barriers and parent's perception of the relevance of the treatment (de Haan et al., 2013), it appears that supporting parents in overcoming barriers to treatment and understanding the relevance of treatment are key targets in order to retain families in parenting programs like PCIT. Services that directly attempt to reduce treatment barriers associated with social disadvantage, through for example the provision of free services, transport, or home visitation, continue to experience high levels of attrition (Damashek, Doughty, Ware, \& Silovsky, 2010; Gomby, Culross, \& Behrman, 1999; Katz et al., 2001), cultivating our hypothesis that empowering caregivers to overcome or be less deterred by their perceived barriers may be more beneficial. Accordingly, in the current trial, a motivational enhancement component was provided prior to families beginning standard 12week PCIT. The motivational enhancement focused on reducing psychological barriers to 
treatment commitment and clarifying the relevance of treatment for parents. Our aim was to reduce attrition and to improve outcomes for families receiving PCIT with the motivational enhancement (M/PCIT) compared with standard 12-week PCIT (S/PCIT) and a supported waitlist condition. Notably, the standard 12-week PCIT format is a time-limited augmentation of the mastery-based protocol, with comparable demonstrated outcomes (Thomas \& ZimmerGembeck, 2012).

\section{Motivational Interviewing}

Motivational Interviewing (MI) is a therapeutic approach attracting increasing recognition for its potential to enhance client engagement in treatment, particularly in areas where clients may be less than "ready, willing, and able" to make behavior change (Hetteme, Steele, \& Miller, 2005, p. 92). Meta-analyses have shown that MI interventions have significant small to medium treatment effects (i.e., behavior change and treatment engagement) when compared to non-active controls. However, these effects were smaller when compared to active treatments (Burke et al, 2003; Lundahl et al, 2010; Vasilaki et al, 2006). Lundahl et al. (2010) concluded that the format of MI interventions (e.g., as an additive component, a stand-alone intervention, or a prelude to another therapy) mattered, and that MI delivered as a prelude to another treatment appeared to be most effective. There was inconclusive evidence for the number of sessions required to ensure sufficient 'dosage' of MI (Lundahl et al, 2010).

\section{Motivational Enhancements to PCIT}

Three previous studies have examined the effectiveness of a motivational enhancement of PCIT among maltreating caregivers. In an effectiveness study conducted in a communitybased organization (Chaffin et al., 2009), PCIT with a prior motivation component was found to yield greater retention to treatment completion when compared with standard PCIT without a motivational component, and when compared with treatment as usual with or 
without a motivation component. In two other studies, motivation-enhanced PCIT was found to reduce future child abuse reports (Chaffin et al., 2004), and subsequently, it was demonstrated that it was the combination of PCIT and motivation, and not either component separately, that produced comparative benefits in child welfare recidivism (Chaffin, Funderburk, Bard, Valle, \& Gurwitch, 2011). These studies implemented a 6-session motivation component in groups, and although retention was improved for parents who reported low or moderate baseline levels of motivation, it was attenuated for parents with high baseline levels of motivation (Chaffin, et al., 2009). However, 22\% of parents who had high baseline levels of motivation and who received standard PCIT without motivational enhancement failed to complete treatment. As such, highly motivated parents also require support to complete treatment. Accordingly, we considered that individual administration of a motivational enhancement as a prelude to PCIT aligns with the client-centered approach of MI, and could better address the unique needs of individual families with varying levels of baseline motivation, thereby reducing attrition even further.

Guided by these findings, we developed three sessions of MI using the same treatment protocol as Chaffin et al. (2004), which were provided as a prelude to PCIT. Lundahl et al. (2010) concluded in their meta-analysis there was inconclusive evidence regarding dosage requirements of MI. Studies included in this meta-analysis reported using between 1 and 18 sessions, with the mean number of MI sessions provided being $2.43($ Median $=2$, Mode $=1)$. Given that the protocol on which our MI sessions were based demonstrated positive outcomes in previous studies (i.e., reduced attrition and child welfare notifications; Chaffin et al., 2009; 2011), the ability to cover content more efficiently in an individual compared to group format, and that three sessions is more than what is typically provided according to Lundahl et al. (2010), we anticipated that this would ensure sufficient dosage to enhance the 
known positive effects of PCIT on parenting and children's behavior (Bagner \& Eyberg, 2007; Thomas \& Zimmer-Gembeck, 2007, 2012; Timmer et al., 2011).

\section{Study Hypotheses}

Our primary hypothesis was that M/PCIT would improve the retention rate, defined as parents completing the full intervention component, when compared with standard PCIT. We also hypothesized that M/PCIT participants would show increases in readiness to change their parenting behaviors from pre- to post-motivational enhancement. Given the robust evidence-base supporting the effectiveness of PCIT for reducing child behavior problems and parenting stress, and improving independent observations of parent-child interactions (Thomas \& Zimmer-Gembeck, 2007), we expected that any form of PCIT (M/PCIT and S/PCIT) would reduce children's externalizing behavior and parents' stress when compared with a supported waitlist condition, as has been previously reported in studies of standard 12week limited PCIT (S/PCIT; Bagner \& Eyberg, 2007; Thomas \& Zimmer-Gembeck, 2012; Timmer et al., 2011). However, because M/PCIT has been shown to be more effective than S/PCIT in reducing child welfare notifications (Chaffin et al., 2011), we also expected that M/PCIT would be more effective in decreasing children's externalizing behaviors and parents' stress compared with S/PCIT.

\section{Method}

\section{Participants}

Participants were 192 Australian caregivers (91.7\% females; $M_{\text {age }}=34.4$ years, $S D=$ 7.3) and their children ( $33.3 \%$ females; $M_{\text {age }}=4.4$ years, $\left.S D=1.2\right)$ from an urban area, referred to a university-based tertiary referral service and research program for parenting support. Referral sources included child protection authorities (27\%), government health services $(25 \%)$, self-referrals (22\%), educational and nongovernment organizations (15\%), or 'other' $(12 \%)$. The referral source for remaining families $(<1 \%)$ was not recorded. Caregivers 
were the target child's biological mother or father $(87 \%)$, foster mother $(6 \%)$, grandparent $(2 \%)$, aunt $(.5 \%)$ or kinship carer $(.5 \%)$. For brevity, the term parent was used to identify all caregivers. Parents reported being married (35\%), in a de-facto relationship (20\%), single $(31.1 \%)$ or divorced/separated $(8 \%)$. Many parents reported having experienced domestic violence $(28 \%)$, and $64 \%$ of those parents reported that their child witnessed the abuse at least once or twice. Parents were predominantly born in Australia (66\%), followed by New Zealand (9\%), and various countries across Europe (6\%), North America (4\%), Asia (3\%), South America (1\%), Africa (1\%), and the Pacific Island Region (.5\%). Four parents (2.1\%) reported being of Australian Aboriginal or Torres Strait Islander descent.

\section{Procedures}

Potential participants and referral sources contacted the parent support program via the telephone. In-person semi-structured interviews with caregivers were scheduled, and caregivers were accepted to the program if children were between the ages of 2.5 and 7 years, and parents experienced at least one of the following: significant levels of parent distress, inappropriate discipline strategies, aggressive parental communication, or child behavior problems. PCIT is contraindicated for sexual abuse perpetrators, thus caregivers were excluded if there was any suspected sexual abuse history based on information from child protection authorities or revealed during the initial interview with parents. The study protocol was discussed with parents and informed consent was obtained during the initial session. Ineligible caregivers were referred to alternative services.

The present study was an extension of a larger trial of PCIT, conducted continuously since 2002 (outcomes of earlier phases of this trial have been previously reported; Thomas \& Zimmer-Gembeck, 2011, 2012). Study approval was obtained from, and conducted in compliance with, the university Human Research Ethics Committee. The present study includes families involved in the program from 2006 to 2013. During this time, participants 
were randomly assigned to PCIT treatment (the original protocol) or a supported waitlist condition that received weekly telephone contact. A randomization ratio of 2(treatment):1(waitlist) was used between 2006 and 2009. Between 2006 and 2009, participants assigned to treatment received standard PCIT (S/PCIT), which involved a maximum of 12 weeks of treatment for each family. Given the evidence of effectiveness of PCIT when data were analyzed in 2009 , the trial continued but a randomization ratio of 5(treatment):1(waitlist) was used between 2009 and 2013, with participants assigned to treatment received motivation enhanced PCIT (M/PCIT), which involved a manualized motivation enhancement protocol in addition to 12 weeks of standard PCIT. Figure 1 shows the flow of S/PCIT, M/PCIT, and waitlist participants through the study between 2006 and 2013. It is important to note that the PCIT protocol has been updated (see McNeil \& Hembree-Kigin, 2010), including changes to the mastery criteria and the use of a backup time-out room. However, to ensure consistency we utilized the same (original) protocol as the foundation across all phases of this trial. In particular, mastery involved demonstrating 25 descriptions and reflections, 15 praises ( 8 of which were labelled), and no more than 3 questions, commands or critical statements in a 5-minute period.

Standard 12-week PCIT. The S/PCIT protocol included two phases. The first phase, child-directed interaction (CDI), involved teaching relationship enhancement skills, and the use of differential reinforcement to shape child behavior (i.e., labeled praise, reflective listening, and ignoring minor misbehavior). The second phase, parent-directed interaction (PDI), focused on teaching parents to effectively provide instructions and confidently practice a discipline protocol for managing non-compliance. Each phase began with a didactic presentation to parents of the specific skills to be practiced for the duration of that phase. The remainder of each phase involved direct coaching of parents while they were interacting with their children to provide the parent with immediate feedback and praise for 
appropriate responses to their child's behavior. Caregivers progressed to the second phase (PDI) when they achieved mastery of CDI skills (McNeil \& Hembree-Kigin, 2010). Overall, parents in the S/PCIT group participated in two assessment sessions (pre- and post-treatment assessment), two didactic information sessions, and a maximum of 12 in vivo coaching sessions.

Motivation enhanced PCIT. Except for the addition of three manualized motivational enhancement sessions, the M/PCIT protocol was the same as S/PCIT. The motivational enhancement sessions were conducted individually with parents, prior to beginning PCIT. These motivation sessions were based on the protocol used by Chaffin et al. (2004), which drew from MI techniques (Miller \& Rollnick, 2002). The motivational enhancement sessions involved watching testimonials from PCIT parent graduates and undertaking decisional balance exercises that evaluated the advantages and disadvantages of harsh physical discipline and parent-generated alternative discipline strategies. These sessions also involved encouraging parents to identify any concerns and goals related to parenting and the parentchild relationship, and exploring parents' commitment to change. Parents in the M/PCIT group participated in three assessment sessions (pre- and post-assessment, and motivation was assessed pre-assessment and post-motivation enhancement), three dyadic motivational enhancement sessions, two didactic information sessions, and up to 12 in vivo coaching sessions.

Waitlist. Participants randomized to the supported waitlist condition were asked to refrain from accessing therapy for child behavior management for the duration of 12 weeks. Parents were phoned weekly by a PCIT therapist to permit a brief discussion of familyrelated concerns. At the end of a 12-week wait period, families were offered S/PCIT or M/PCIT depending on the PCIT program provided in the Centre at that time. Data collection from these families while they were in treatment are not included in the present study. 
Training and treatment integrity. Fourteen masters or doctoral level therapists (registered as psychologists or psychologist interns) implemented the intervention between 2006 and 2013, with no more than five therapists working at any one time. All therapists were trained and supervised by one senior PCIT psychologist (second author; masked for review) who was trained by the PCIT CAARE team in Sacramento, USA. The second author had over 5 years of experience solely providing PCIT, and was accredited to train and supervise others. Therapists underwent extensive training over a period of approximately 12 months, including observation of the senior therapist, followed by co-facilitation, practice under direct supervision, and then independent practice. The senior therapist was available during all hours of operation for consultation, and provided weekly supervision of PCIT implementation and fidelity checks via individual consultation and observations of PCIT sessions both when requested and at random. Between 2009 and 2013, eight therapists provided M/PCIT, and group supervision was held fortnightly to ensure adherence to the manualized motivation enhancement component. Thus, maintaining treatment fidelity was a priority, but not systematically assessed. This study was conducted continuously from 2006 to 2013, with a brief transition from S/PCIT to M/PCIT in 2009. No systematic changes in the context of the treatment program were identified between treatment phases that may have contributed to differences in treatment engagement or outcomes over time.

Data collection. Parent-report measures were provided during the initial interview, completed at home, and returned the following session when randomization occurred. Postassessment data were collected after completion of either the S/PCIT or M/PCIT protocol, or after 12 weeks for waitlist participants.

\section{Measures}

Child externalizing and internalizing behaviors. The parent report versions of the Child Behavior Checklist (CBCL; Achenbach, 1991; Achenbach \& Rescorla, 2000, 2001) 
and the Eyberg Child Behavior Inventory (ECBI, Eyberg \& Pincus, 1999) were used to assess child internalizing and externalizing symptoms. The CBCL is a behavioral rating scale for children aged 1.5 to 18 years. The scale describes a range of behavioral and emotional problems, and requires responders to indicate the response that best describes their child $(0=$ not true, $1=$ somewhat true, $2=$ very true). Items are summed to produce internalizing and externalizing subscale raw scores, and raw scores are then converted to T-scores, which have a mean of 50 and a SD of 10 . T-scores are used to enable comparisons across different versions of the CBCL. The borderline clinical range is represented by a T-score between 60 and 63 , and a T-score of 64 or above is considered to be within the clinical range. In the present study, Cronbach's $\alpha$ for externalizing symptoms was .87 for female caregivers and .82 for male caregivers, and Cronbach's $\alpha$ for internalizing symptoms was .85 for female caregivers and .84 for male caregivers.

The ECBI presents respondents with a range of disruptive child behaviors, and requires parents to report the frequency of each behavior (ECBI Intensity) and the extent to which parents found the behaviors to be problematic (ECBI Problem). Response options for ECBI Intensity range from 1 (never) to 7 (always), and summing these scores forms the intensity subscale score. For the problem subscale, parents indicate on a dichotomous yes/no scale whether each behavior is problematic, and summing the endorsed items forms the problem subscale score. An intensity score of 132 and a problem score of 15 indicate clinical problems in children aged 2 to 12 years (Eyberg \& Pincus, 1999). In the present study, Cronbach's $\alpha$ for ECBI Intensity was .94 and .93 for females and males, respectively, and for ECBI Problem it was .90 and .88 for females and males, respectively.

Parent stress. The Parenting Stress Inventory, Third Edition (PSI-3; Abidin, 1995) was used to evaluate the degree of parent stress within the parent-child system. Composite scores for the child and parent stress domains are formed from 101 items. Response options for 90 
of the items range from 0 (strongly disagree) to 5 (strongly agree), 11 items have specific multiple choice options, and the remaining 19 items involve a dichotomous yes/no response regarding particular life stressors. Summing items forms subscale scores, with high scores on the parent stress and child stress domains indicating that parental characteristics and child characteristics, respectively, are a significant source of stress in the parent-child relationship. A score $\geq 148$ for the parent domain, and $\geq 116$ for the child domain, indicate parent stress that is $\geq 85$ th percentile. Cronbach's $\alpha$ for the parent stress domain was .93 for female caregivers and .94 for male caregivers, and for the child stress domain it was .93 for female caregivers and .95 for male caregivers.

Readiness for change (motivation). The Readiness for Parenting Change Scale (Chaffin et al., 2009) was used to assess participant motivation to change (23 items, e.g., "I am ready to change the way I discipline my child"). Responses ranged from 1 (strongly disagree) to 5 (strongly agree), and composite scores were formed by averaging the items. Cronbach's $\alpha$ was .87 for females and .94 for males. A higher score indicated greater readiness to change.

\section{Overview of Analyses}

Prior to examining attrition rates and outcomes, descriptive statistics (e.g., means and standard deviations), one way analyses of variance (ANOVA), and $\chi^{2}$ tests were conducted to compare the four groups (M/PCIT, S/PCIT, waitlist 2006 - 2009, waitlist 2009 - 2013) at pre-assessment on all measures. The two waitlist groups were combined into a single group as no differences between these groups was found in outcome measures at pre- or postassessment. Next, change in motivation from pre-assessment to post-motivational enhancement in the M/PCIT group was examined using a repeated measures $t$-test.

To evaluate the impact of motivational enhancement on retention, four strategies were used. First, a $\chi^{2}$ test was used to compare attrition between the M/PCIT and S/PCIT groups. 
Second, survival analysis was used to compare the rate of attrition over the weeks of treatment or waitlist. Third, M/PCIT caregivers were categorized into groups and retention was compared between groups. This involved using $\chi^{2}$ tests to compare the retention rate between (a) caregivers' high (top 50\%) in readiness to change and those low (bottom 50\%) in readiness to change, (b) caregivers who were in two bands of readiness to change scores at pre-assessment (i.e., 2.01-3 or 3.01-4), which captured all scores, and (c) caregivers who were in two bands of readiness to change when assessed post-motivational enhancement (i.e., 3.01-4, and 4.01-5), which captured all scores. Fourth, readiness to change scores at preassessment and post-motivation enhancement, and change in readiness to change scores from pre-assessment to post-motivation enhancement, were examined to identify a criterion cutoff which predicted retention at a rate $\geqslant 85 \%$.

To compare treatment outcomes between the three groups, 3 (Group: M/PCIT vs. S/PCIT vs. combined waitlist) $\times 2$ (Time: pre-assessment vs. post-assessment) mixed factorial ANOVAs were used. Due to the pre-assessment difference between S/PCIT and $\mathrm{M} / \mathrm{PCIT}$ in proportion of referrals from child protection authorities, all outcomes were also submitted to a 2 (Referral source: Child protection authorities vs. other) $\times 3$ (Group: M/PCIT vs. S/PCIT vs. combined waitlist) $\times 2$ (Time: pre-assessment vs. post-assessment) mixed factorial ANOVA. To provide an indication of the clinical significance of the findings, postassessment scores for each group are compared to measurement norms. Moreover, a Reliable Change Index (RCI) was calculated for each group on each outcome measure (RCI $\geq 1.96$ is statistically significant $p<.05$; Jacobson \& Truax, 1991).

There have been many discussions about the importance of minimizing missing data and selecting the right method for dealing with missingness and dropout (e.g., Graham, 2009; Little \& Rubin, 2002). In the present study, we first focused on participants who completed both pre- and post-assessments finding that $1.8 \%$ of their data were missing. According to 
Little's MCAR test (Little, 1998), this missingness was completely at random. To manage this minimal missing data and retain all these participants in the 'completer' analyses, total scores were calculated based on the completed items when a participant was missing only a single item. When all items on a scale were missing, multiple imputation was used to estimate total scores (Graham, 2009). All Time $\times$ Group effects and simple effects significant for the original dataset were also significant in the pooled results from multiple imputation. Thus, the results reported here are the pooled results from five imputed datasets.

Intention-to-treat (ITT) analyses were also conducted using the last data point carried forward method (LOCF; Gupta, 2011; Waters et al., 2014), where treatment and waitlist participants who were missing the entire post-assessment were assigned the same scores at post-assessment as they had at pre-assessment. ITT has been suggested as best practice to manage missingness and dropout (Gupta, 2011). Although there remains discussion about the best ITT method to use and no firm guidelines regarding the best method (e.g., see the Consort Guidelines regarding transparent reporting of randomized control trials; Schulz, Altman, \& Moher, 2010), LOCF was used in the present study because it has been described as the best method for study designs with two repeated assessments (pre/post) of both the treatment and waitlist groups (Gupta, 2011). It is important to note, however, that LOCF is a conservative approach to evaluating treatment outcomes by assuming no difference between pre- and post-assessment. However, this method also depends on the assumption that no participant declined in his or her functioning (e.g., no child increased in externalizing symptoms from pre- to post-assessment), which we believed was an assumption supported by the literature showing the general effectiveness of PCIT (Chaffin et al., 2009; Thomas \& Zimmer-Gembeck, 2007). These issues should be kept in mind when interpreting the effect sizes from the ITT analyses.

\section{Results}




\section{Means, Standard Deviations, and Treatment Group Differences at Pre-Assessment}

There were no differences between the four groups (S/PCIT, M/PCIT, $2006-2009$ waitlist; 2009 - 2013 waitlist) in mean child age, mean parent age, or parent education level. There were also no group differences in the proportion of children who were male vs. female, whether or not the child lived away from parents, parental report of domestic violence (yes or no), sociocultural background of parents, parent employment status, income, or marital status. There was a significant difference in referral source, $\chi^{2}(15, N=191)=27.46, p=.03$, whereby a greater proportion of S/PCIT caregivers (36.1\%) than M/PCIT caregivers (18.4\%) were referred from child protection authorities $(p<.05)$. However, Referral source $\times$ Group $\times$ Time interactions on all outcomes were not significant, $F$ ranged from 0.04 to 2.03, $p$ ranged from .14 to .96 . Moreover, there were no differences in dropout according to referral source, $\chi^{2}(5, N=191)=10.43, p=.06$, nor were there differences in pre-assessment motivation according to referral source, $F(5,82)=1.52, p=.19$. No differences were found at preassessment on any outcome variable, including child internalizing and externalizing behavior or parent stress, $F$ ranged from 0.46 to $1.91, p$ ranged from .13 to .71 . As there were no group differences in outcome measures or demographics at pre-assessment and post-assessment for participants in the supported waitlist condition, the two waitlist groups were combined to form a single waitlist group for comparison to the two treatment conditions.

\section{Motivational Enhancement, Readiness to Change and Retention}

As expected, there was a significant increase in readiness to change in M/PCIT caregivers from pre-assessment $(M=3.1, S D=0.2)$ to post-motivational enhancement $(M=$ 4.2, $S D=0.4), F(1,39)=448.61, p<.001, \eta_{\text {partial }}^{2}=.92$. Reliable change in motivation was demonstrated in $97 \%$ of caregivers.

Contrary to our hypothesis, there was no difference between the two treatment groups in attrition rate at the end of 12 weeks, $\chi^{2}(1, N=138)=1.58, p=.22$. The attrition rate was 
$41.6 \%$ in M/PCIT, and $31.1 \%$ in S/PCIT. Survival analysis revealed that the rate of attrition across weeks in treatment did not differ between S/PCIT and M/PCIT, Wilcoxon $=2.07, d f=$ $1, p=.15$ (see Figure 2).

When M/PCIT caregivers were categorized into either a high or low motivation group based on being above or below the median score of pre-treatment readiness to change, respectively, attrition was significantly lower for caregivers who reported high pre-treatment motivation (25\% attrition) compared with caregivers who reported low motivation $(57.5 \%$ attrition), $\chi^{2}(1, N=76)=8.21, p=.004$. Survival analysis confirmed that the rate of attrition was significantly earlier and higher overall among caregivers' low in motivation at preassessment $(M d n$ survival time $=9.5$ sessions $)$ compared to caregivers high in motivation $(M d n$ survival time $=12.0$ sessions; Wilcoxon $=6.62, d f=1, p=.01)$. Figure 2 illustrates survival patterns in the two active treatment groups (S/PCIT and M/PCIT), and in the high and low pre-assessment motivation groups (subgroups of M/PCIT). Notably, all M/PCIT caregivers reported a motivation level after the three motivational sessions that was above the median value reported prior to the motivational sessions.

We next examined the proportion of caregivers who completed PCIT within particular bands of readiness to change scores. Given the range of scores at pre-assessment (2.36 to 3.68), we compared caregivers in two bands of scores (3.00 or lower vs. 3.01 or higher), finding that $71 \%$ of parents with a pre-assessment score in the higher band completed PCIT treatment, as compared to $44 \%$ of parents with a readiness to change score in the lower band, $\chi^{2}=5.95, p=.02$. At the post-motivation enhancement assessment, the range of motivation scores was 3.29 to 4.86. Thus, we compared retention between two bands (4.00 or lower and 4.01 or higher), finding that there was no significant difference in retention between these two groups (67\% in the lower group and $84 \%$ in the higher group), $\chi^{2}=1.62, p=.26$.

Finally, we identified a criterion cutoff score, which when exceeded, was found to 
predict retention at a rate greater than $85 \%$. Specifically, $86 \%$ of caregivers who reported an increase of 1.24 or more from pre-assessment to post-motivation enhancement in readiness to change were retained to completion, compared to $73 \%$ of those who reported less of an increase. Notably, retention of caregivers who reached this criterion was not significantly higher than for those who did not $(p=.45)$, however this level of change in motivation represents a criterion for achieving $\geqslant 85 \%$ retention none-the-less.

\section{Treatment Outcomes}

Child externalizing and internalizing symptoms. Analyses of the CBCL subscales identified a significant main effect of Time on child externalizing and internalizing symptoms. However, each of these main effects was modified by a significant Group $\times$ Time interaction (Table 1 presents all Group $\times$ Time interaction effects). Simple effect analyses were used to investigate changes in child behavior symptoms from pre- to post-assessment. Child externalizing symptoms declined from pre- to post-assessment in all groups, with the greatest declines observed in the M/PCIT $\left(95 \% \mathrm{CI}_{\text {diff }}=5.46,10.22\right)$ and S/PCIT treatment groups $\left(95 \% \mathrm{CI}_{\text {diff }}=5.89,10.95\right)$. The decline in the waitlist group was smaller, but

significant $\left(95 \% \mathrm{CI}_{\mathrm{diff}}=0.39,5.44\right), F(2,122)=5.70, p=.004, \eta_{\text {partial }}^{2}=0.09$. When the two treatment groups were compared in a 2 (Group: M/PCIT vs. S/PCIT) $\times 2$ (Time: preassessment vs. post-assessment) mixed factorial ANOVA, the Group $\times$ Time interaction was not significant for externalizing symptoms, $F(1,83)=0.10, p=.75, \eta_{\text {partial }}^{2}=0.001$. The proportion of caregivers who reported their child's externalizing symptoms in the normal range at post-assessment was $64.4 \%$ for M/PCIT, $72.5 \%$ for S/PCIT, and $50 \%$ in the waitlist group. The proportion of caregivers who reported reliable change in child externalizing symptoms was $47 \%$ for M/PCIT, $48 \%$ for S/PCIT, and $15 \%$ in the waitlist group.

Internalizing symptoms significantly declined in the M/PCIT $\left(95 \% \mathrm{CI}_{\text {diff }}=1.81,7.00\right)$ and S/PCIT groups $\left(95 \% \mathrm{CI}_{\text {diff }}=4.33,9.83\right)$, but not in the waitlist group $\left(95 \% \mathrm{CI}_{\text {diff }}=-1.25\right.$, 
4.25), $F(2,122)=4.04, p=.02, \eta_{\text {partial }}^{2}=0.06$. When the two treatment groups were compared in a 2 (Group: M/PCIT vs. S/PCIT) $\times 2$ (Time: pre-assessment vs. post-assessment) mixed factorial ANOVA, the Group $\times$ Time interaction for child internalizing symptoms was not significant, $F(1,83)=1.93, p=.17, \eta_{\text {partial }}^{2}=0.02$. At post-assessment, the proportion of caregivers who reported their child's internalizing symptoms in the normal range was $77.8 \%$ for M/PCIT, $90 \%$ for S/PCIT, and $72.5 \%$ in the waitlist group. The proportion of caregivers who reported reliable change was $13 \%$ for M/PCIT, $30 \%$ for S/PCIT, and $10 \%$ in the waitlist group.

Child behavior intensity and problems. Analyses of the ECBI subscales identified a significant main effect of Time, as well as Group $\times$ Time interactions, on child behavior intensity and child behavior problems. Child behavior intensity declined in all groups, with the greatest declines evident in the M/PCIT $\left(95 \% \mathrm{CI}_{\text {diff }}=24.49,39.96\right)$ and S/PCIT treatment groups $\left(95 \% \mathrm{CI}_{\text {diff }}=18.82,35.23\right)$, compared with the waitlist group $\left(95 \% \mathrm{CI}_{\text {diff }}=2.62\right.$, $19.03), F(2,122)=7.53, p=.001, \eta_{\text {partial }}^{2}=0.11$. At post-assessment, $60 \%$ of M/PCIT caregivers, $65 \%$ of S/PCIT caregivers, and $40 \%$ of the waitlist caregivers reported their child's behavior intensity as being within the normal range. The proportion of caregivers who reported reliable change was $56 \%$ for M/PCIT, $65 \%$ for S/PCIT, and $28 \%$ in the waitlist group.

Child behavior problems declined in M/PCIT $\left(95 \% \mathrm{CI}_{\text {diff }}=6.89,10.98\right)$ and S/PCIT treatment groups $\left(95 \% \mathrm{CI}_{\text {diff }}=6.58,10.92\right)$, but not in the waitlist group $\left(95 \% \mathrm{CI}_{\text {diff }}=-0.10\right.$, $4.25), F(2,122)=12.97, p=<.001, \eta_{\text {partial }}^{2}=0.18$. When the two treatment groups were compared in 2 (Group: M/PCIT vs. S/PCIT) $\times 2$ (Time: pre-assessment vs. post-assessment) mixed factorial ANOVAs, the Group $\times$ Time interaction was not significant for child behavior intensity, $F(1,83)=0.81, p=.37, \eta_{\text {partial }}^{2}=0.01$, or for child behavior problems, $F(1,83)=0.01, p=.91, \eta_{\text {partial }}^{2}<0.01$. The proportion of caregivers who reported their 
child's behavior problems as being in the normal range at post-assessment was $73.3 \%$ for M/PCIT, $70 \%$ for S/PCIT, and 50\% for the waitlist group. The proportion of caregivers who reported reliable change was $58 \%$ for M/PCIT, $53 \%$ for S/PCIT, and $15 \%$ in the waitlist group.

Parent stress. Parent stress pertaining both to the child and to the parenting role declined over time, but these declines were modified by Group $\times$ Time interactions. Parent stress due to the child declined between pre- and post-assessment in the M/PCIT $\left(95 \% \mathrm{CI}_{\text {diff }}=\right.$ $12.22,21.69)$ and S/PCIT treatment groups $\left(95 \% \mathrm{CI}_{\mathrm{diff}}=12.76,22.79\right)$, but there was not a significant change in parents' stress due to the child in the waitlist group $\left(95 \% \mathrm{CI}_{\text {diff }}=-3.24\right.$, $6.80), F(2,122)=12.83, p<.001, \eta_{\text {partial }}^{2}=0.17$. At post-assessment, the proportion of caregivers who reported parent stress pertaining to the child that was below the measure's $85^{\text {th }}$ percentile was $46.7 \%$ of M/PCIT, $46.5 \%$ for S/PCIT, and $37.5 \%$ for the waitlist. The proportion of caregivers who reported reliable change was $44 \%$ for M/PCIT, $43 \%$ for S/PCIT, and $10 \%$ in the waitlist group.

Parent stress relating to the parenting role between pre- and post-assessment in the M/PCIT $\left(95 \% \mathrm{CI}_{\text {diff }}=3.61,13.46\right)$ and S/PCIT treatment groups $\left(95 \% \mathrm{CI}_{\mathrm{diff}}=7.89,18.33\right)$, but there was not a significant change in parents' stress due to the child in the waitlist group $\left(95 \% \mathrm{CI}_{\text {diff }}=-2.13,8.31\right), F(2,122)=3.62, p=.03, \eta_{\text {partial }}^{2}=0.06$. When the two treatment groups were compared in 2 (Group: M/PCIT vs. S/PCIT) $\times 2$ (Time: pre-assessment vs. postassessment) mixed factorial ANOVAs, the Group $\times$ Time interaction was not significant for parenting stress relating to the child, $F(1,83)=0.05, p=.82, \eta_{\text {partial }}^{2}=0.001$, or to the parenting role, $F(1,83)=1.56, p=.22, \eta_{\text {partial }}^{2}=0.02$. At post-assessment, $66.7 \%$ of M/PCIT caregivers, $80 \%$ of S/PCIT caregivers, and $60 \%$ of waitlist caregivers reported parent stress pertaining to the parenting role below the $85^{\text {th }}$ percentile. The proportion of caregivers who 
reported reliable change was $20 \%$ for M/PCIT, $35 \%$ for S/PCIT, and $15 \%$ in the waitlist group.

\section{Intent to Treat Analyses: Treatment Group Differences on Outcome Measures}

Table 2 presents all ITT Group $\times$ Time interaction effects. ITT analyses produced comparable results to those described above.

\section{Discussion}

We examined whether adding a 3-session individualized motivational component as a prelude to a standard 12-week PCIT protocol reduced attrition and improved outcomes for multiproblem treatment-seeking families in comparison to a similar group of clients receiving no motivational enhancement. The findings suggest that an individually delivered motivational component can significantly increase parents' readiness to change prior to beginning the first session of PCIT, but it may not lower the attrition rate or enhance treatment outcomes compared with standard 12-week PCIT. Overall, we found no significant difference in the attrition rate or pattern of attrition when we compared families receiving PCIT with or without the motivational enhancement (M/PCIT vs. S/PCIT). Three previous studies have examined the effect of adding a motivational component to standard PCIT on child abuse recidivism (Chaffin et al., 2004; 2011) and on attrition (Chaffin et al., 2009). When comparing our attrition rates to this previous study (Chaffin et al., 2009), attrition in S/PCIT (31.1\%) compared favorably to the standard orientation with PCIT in this previous study (38\%), but our M/PCIT attrition rate of $41.6 \%$ was much higher than the motivation orientation with PCIT in this previous study $(15 \%)$.

Notably, Chaffin et al. (2009) reported more attrition from parents who received the motivation orientation when motivation was already high. While we could not evaluate whether attrition differed for highly motivated parents according to the treatment they received (i.e., M/PCIT or S/PCIT) as the motivation questionnaire was not completed by the 
families assigned to S/PCIT in the present study, we were able to evaluate whether level of motivation among families in M/PCIT was associated with treatment retention. Specifically, it was found that parents who reported higher (above the median) initial motivation were less likely to drop out of treatment, and showed a slower rate of drop out (i.e., they remained in treatment longer), compared with parents who reported lower initial motivation. Moreover, parents with a readiness to change score of 3 or higher prior to treatment were significantly more likely to remain in treatment than parents with a score less than 3. However, no such conclusions could be made regarding readiness to change scores post-motivation enhancement. On the other hand, a criterion level of improvement of $\geq 1.24$ in readiness to change from pre-assessment to post-motivation enhancement predicted retention at a rate of $\geq 85 \%$. As such, parents' initial motivation (in particular, a readiness to change score less than 3) was indicative of subsequent attrition, yet motivational interviewing was successful in improving parents' readiness to change parenting practices, and there was some evidence that enhancing motivation was protective of premature attrition to the extent that caregivers achieved a high degree of change in readiness to change after motivation enhancement. It may be that an emphasis on ensuring sufficient growth in motivation is required prior to progressing to the coaching phase of PCIT, to prevent treatment dropout.

Alternatively, the discrepancy between attrition for MI caregivers in the present study compared to the prior study of attrition by Chaffin and colleagues (2009) may be the result of differences in methodology. Specifically, this previous study provided six motivational enhancement sessions in a group format, for caregivers referred from child welfare agencies. In contrast, in the present study, the same MI activities were delivered in three sessions, individually, and caregivers were referred from a range of sources (18.4\% from child welfare agencies). As such, although we anticipated that individually delivered motivational enhancement may be more beneficial due to the alignment of individualized sessions with the 
client-centered approach of MI, it may be that MI delivered in a group format, which permits sharing of experiences and ideas, is superior for motivating and engaging caregivers, thereby reducing attrition. It may also be that caregivers referred for parenting support by child welfare agencies (of whom comprised the entire sample in the previous study but not the present study) may be more amenable to the positive effects of MI, due to the potential consequences of failing to attend mandated treatment. Moreover, previous studies of motivation-enhanced PCIT found that motivation improved among caregivers who received MI as well as among caregivers who received a standard orientation to services, however, gains in motivation were greater among MI caregivers. In the present study, a significant increase in motivation was observed among M/PCIT caregivers, and after motivation enhancement all M/PCIT caregivers reported motivation above the value that was used to differentiate high from low motivation caregivers. However, we were not able to examine change in motivation among parents who received S/PCIT, and as such could not determine whether enhanced motivation was observed among M/PCIT compared to S/PCIT caregivers. Accordingly, it may be that M/PCIT caregivers did not experience increases in motivation beyond that experienced by S/PCIT caregivers, resulting in comparable levels of attrition and treatment outcomes between these two treatment groups.

Notably, all of the attrition rates reported in the present study fall below the means reported by de Haan and colleagues' meta-analysis (2013) of 50\% for an effectiveness study and $44 \%$ for studies defining attrition as non-completion of sessions. Our study fulfilled both these criteria; our inclusion criteria were broad and our exclusion criteria narrow, and our definition of attrition was non-completion of the treatment protocol. Thus, it may be that our attrition rates were reaching the floor for an active and intensive treatment like PCIT, and motivational enhancement may not have been enough or the right approach for reducing them even further. 
PCIT, either S/PCIT or M/PCIT, was effective at reducing caregiver-reported child behavior problems and parent stress pertaining to the child, compared with waitlist, upholding the robust intervention outcomes of PCIT. No differences were noted in ITT analyses. Overall, these outcomes are similar to the results of a recent meta-analysis of PCIT, whereby standard PCIT surpassed modified PCIT (Thomas, Abell, Webb, Avdagic, \& Zimmer-Gembeck, 2016), suggesting that when researchers and practitioners add more to an already efficacious intervention the outcomes are not necessarily better.

The current study builds on previous data, was embedded within a well-established PCIT research cohort, and was implemented by highly skilled therapists. To target the unique needs of individual families we integrated effective MI strategies (decisional balance exercises, testimonials, and evoking change talk through looking forward) into a manualized therapeutic module and implemented it within a PCIT framework. However, there are some limitations to this approach. In a meta-analysis of clinical trials of MI interventions, manualized protocols tended to produce small effect sizes (Hetteme, Steele, \& Miller, 2005). To explain this finding, the authors provided an example of where adherence to the manual may have inadvertently violated core MI principals, by completing a task that elicited resistance in some clients who were less ready for change (Hetteme et al., 2005; Miller, Yahne, \& Tonigan, 2003). It is possible that in our attempt to standardize the motivational component of our study, we may not have met the needs of all participants. Future research might consider incorporating greater flexibility in the use of motivational techniques, consistent with the person-centered principals of MI. On the other hand, MI delivered in groups prior to PCIT produced reductions in attrition and child welfare reports (Chaffin et al., 2004; 2009; 2011), while individual MI in the present study did not. Therefore, it may be that it is the group format of MI that is effective at maintaining client engagement in relation to PCIT. 
It is notable that the present study utilized a 12-week PCIT protocol as a basis for both treatment groups, in accordance with Chaffin et al $(2004 ; 2009 ; 2011)$, and although comparable outcomes to the original time-variable mastery based protocol have been previously demonstrated (Thomas \& Zimmer-Gembeck, 2012), the generalizability of the present results to results based on the original protocol should be considered. In particular, the inherent provision of a designated end point in standard 12-week PCIT, but not in timevariable mastery based PCIT, may have an effect of reduced attrition. In addition, caregivers in this study were predominantly born in Australia or New Zealand, potentially limiting the generalizability of results to families from other cultural backgrounds, including Australia's First Peoples. Finally, the lack of systematic assessment of treatment fidelity and the fact that all data on motivation and treatment outcomes were self-reported by the parents represent further study limitations, which may reduce the validity of the results. Notably, however, our previous trials of PCIT demonstrated improvements in observed parent behaviors (i.e., reduced negative verbalizations, and increased positive verbalizations and maternal sensitivity) consistent with improvements of parents' self-reports of stress and children's behavior (Thomas \& Zimmer-Gembeck, 2011, 2012).

Research has demonstrated that even when programs specifically focus on maintaining families, problematic levels of attrition are reported (41\%; Katz et al., 2001). As such, continued research attention is required. Motivation is one factor used to engage individuals to change their behaviors, with Chaffin et al. (2009) finding that motivational enhancement improved retention of mothers attending PCIT, but not those attending a didactic parent group, with improved retention only observed in mothers who initially reported low levels of motivation. In the current study, the individually delivered MI component increased caregivers' readiness to change parenting behaviors, but this did not translate into greater retention. However, more than $85 \%$ of caregivers who demonstrated a high degree of 
improvement in readiness to change completed treatment. As such, achievement of a criterion level of improvement in motivation to change parenting practices could be examined in future research as a prerequisite for initiating PCIT coaching in order to reduce attrition. On the other hand, there may be other factors in addition to and associated with motivation, that are contributing to attrition. In a meta-analysis of attrition in child and adolescent mental health interventions, de Haan and colleagues (2013) noted that a range of factors have been reported as predictive of attrition, including factors relating to the child, parent, household, therapist, treatment, and study design. Notably, the large number of predictors studied and diversity in study design makes conclusions about the major contributors difficult. Continued research specifically focused on examining and targeting multiple indicators of attrition in parenting programs is needed.

The findings of this study add to the burgeoning literature showing that PCIT is an effective intervention for improving parenting practices, and reducing child behavior problems and parental stress among multiproblem and maltreating families (Batzer, Berg, Godinet, Stotzer, 2015; Chaffin et al., 2011; Thomas \& Zimmer-Gembeck, 2007, 2011; Timmer et al., 2011). However, attrition from PCIT was still higher than ideal even with a motivational enhancement. Future research is needed to understand the best strategies to use to assist families so that all who access services can participate fully in evidence-based parenting interventions. 


\section{References}

Abidin, R. R. (1995). Parenting stress index third edition: Professional manual. Lutz, FL: Psychological Assessment Resources, Inc.

Achenbach, T. M. (1991). Integrative guide for the 1991 CBCL/4-18, YSR and TRF profiles. Burlington: Department of Psychiatry, University of Vermont.

Achenbach, T. M., \& Rescorla, L. A. (2000). Manual for the ASEBA preschool forms and profiles. Burlington, VT: University of Vermont Department of Psychiatry.

Achenbach, T. M., \& Rescorla, L. A. (2001). Manual for the ASEBA school-age forms and profiles. Burlington, VT: University of Vermont, Research Center for Children, Youth, and Families.

Bagner, D. M., \& Eyberg, S. M. (2007). Parent-Child Interaction Therapy for disruptive behavior in children with mental retardation: A randomized controlled trial. Journal of Clinical Child \& Adolescent Psychology, 36, 418-429.

doi:10.1080/15374410701448448

Bagner, D. M., \& Graziano, P. A. (2013). Barriers to success in parent training for young children with developmental delay: The role of cumulative risk. Behavior Modification, 37, 356-377. doi:10.1177/0145445512465307

Batzer, S., Berg, T., Godinet, M. T., \& Stotzer, R. L. (2015). Efficacy or chaos? Parent-Child Interaction Therapy in maltreating populations: A review of research. Trauma Violence Abuse. doi:10.1177/1524838015620819

Burke, B. L., Arkowitz, H., \& Menchola, M. (2003). The efficacy of motivational interviewing: A meta-analysis of controlled clinical trials. Journal of Consulting and Clinical Psychology, 71, 843-861. doi:10.1037/0022-006X.71.5.843

Chaffin, M., Funderburk, B., Bard, D., Valle, L. A., \& Gurwitch, R. (2011). A combined motivation and Parent-Child Interaction Therapy Package reduces child welfare 
recidivism in a randomized dismantling field trial. Journal of Consulting and Clinical Psychology, 79, 84-95. doi:10.1037/a0021227.

Chaffin, M., Silovsky, J. F., Funderburk, B., Valle, L. A., Brest an, E. V., Balachova, T., Jackson, S., Lensgraf, J., \& Bonner, B. L. (2004). Parent-Child Interaction Therapy with physically abusive parents: Efficacy for reducing future abuse reports. Journal of Consulting and Clinical Psychology, 72, 500-510. doi: 10.1037/0022-006X.72.3.500

Chaffin, M., Valle, L. A., Funderburk, B., Gurwitch, R., Silovsky J., Bard, D., McCoy, C., \& Kees, M. (2009). A motivational intervention can improve retention for PCIT for lowmotivation child welfare clients. Child Maltreatment, 14, 356-368. doi:10.1177/1077559509332263.

Damashek, A., Doughty, D., Ware, L., \& Silovsky, J. (2010). Predictors of client engagement and attrition in home-based child maltreatment prevention services. Child Maltreatment, 16, 9-20. doi:1177/1077559510388507

de Graaf, I., Speetjens, P., Smit, F., de Wolff, M., \& Tavecchio, L. (2008). Effectiveness of the Triple P Positive Parenting Program on behavioral problems in children: A metaAnalysis. Behavior Modification, 32, 714-735. doi:10.1177/0145445508317134

de Haan, A. M., Boon, A. E., de Jong, J. T. M., Hoeve M., \& Vermeiren, R. R. J. M. (2013). A meta-analytic review on treatment dropout in child and adolescent outpatient mental health care. Clinical Psychology Review, 33, 698-711. doi:10.1016/j.cpr.2013.04.005

Dishion, T. J., \& Patterson, G. R. (2006). The development and ecology of antisocial behavior in children and adolescents. In D. Cicchetti \& D. J. Cohen (Eds.). Developmental psychopathology, volume three. Risk, disorder, and adaptation (pp. 503-541). New Jersey: John Wiley \& Sons Inc. 
Eyberg, S. M., \& Pincus, D. (1999). Eyberg Child Behavior Inventory and Sutter-Eyberg Student Behavior Inventory: Professional manual. Odessa, FL: Psychological Assessment Resources.

Eyberg, S. M., \& Robinson, E. A. (1982). Parent-child interaction training: Effects on family functioning. Journal of Clinical Child Psychology, 11, 130-137. doi: $\underline{10.1080 / 15374418209533076}$

Fernandez, M. A., \& Eyberg, S. M. (2009). Predicting treatment and follow-up attrition in Parent-Child Interaction Therapy. Journal of Abnormal Child Psychology, 37, 431441. doi:10.1007/s10802-008-9281-1

Gomby, D. S., Culross, P. L., \& Behrman, R. E. (1999). Home visiting: recent program evaluations-Analysis and recommendations. The Future of Children, 9, 5-25.

Goodman, A., Joyce, R., \& Smith, P. (2011). The long shadow cast by childhood physical and mental problems on adult life. Proceedings of the National Academy of Sciences, 108, 6032-6037. doi:10.1073/pnas.1016970108

Graham, J. W. (2009). Missing data analysis: Making it work in the real world. Annual Review of Psychology, 60, 549.576.

Gupta, S. K. (2011). Intention-to-treat concept: A review. Perspectives in Clinical Research, 2, 109-112.

Hetteme, J., Steele, J., \& Miller, W. R. (2005). Motivational interviewing. Annual Review of Clinical Psychology, 1, 91-111. doi:10.1146/annurev.clinpsy.1.102803.143833

Hiscock, H., Roberts, G., Efron, D., Sewell, J. R., Bryson, H. E., Price, A. M. H., Oberklaid, F., South, M., \& Wake, M. A. (2011). Children attending a paediatricians study: A national prospective audit of outpatient practice from the Australian paediatric research network. The Medical Journal of Australia, 194, 392-397. 
Holden, G. H., Lavigne, V. V., \& Cameron, A. M. (1990). Probing the continuum of effectiveness in parent training: Characteristics of parents and preschoolers. Journal of Clinical Child Psychology, 19, 1, 2-8. doi:10.1207/s15374424jccp1901_1

Jacobson, N. S., \& Truax, P. (1991). Clinical significance: A statistical approach to defining meaningful change in psychotherapy research. Journal of Consulting and Clinical Psychology, 59, 12-19.

Katz, K. S., El-Mohandes, A., Johnson, D. M., Jarrett, M., Rose, L., \& Cober, M. (2001). Retention of low income mothers in a parenting intervention study. Journal of Community Health, 26, 203-218. doi:10.1023/A:1010373113060

Lanier, P., Kohl, P. L., Benz, J., Swinger, D., Moussette, P., \& Drake, B. (2011). ParentChild interaction therapy in a community setting: Examining outcomes, attrition, and treatment setting. Research on Social Work Practice, 21, 689-698. doi:10.1177/1049731511406551

Little, R. J. A. (1988). A test of missing completely at random for multivariate data with missing values. Journal of the American Statistical Association, 83, 1198-1202.

Little, R. J. A., \& Rubin, D. B. (2002). Statistical analysis with missing data ( $2^{\text {nd }}$ edition). New York: Wiley.

Lundahl, B. W., Kunz, C., Brownell, C., Tollefson, D., \& Burke, B. L. (2010). A metaanalysis of motivational interviewing: Twenty-five years of empirical studies. Research on Social Work Practice, 2, 137-160. doi:10.1177/1049731509347850.

McNeil, C. B., \& Hembree-Kigin, T. L. (2010). Parent-Child Interaction Therapy ( $2^{\text {nd }}$ ed.). New York, NY: Springer.

Miller, W. R., \& Rollnick, S. (2002). Motivational Interviewing: preparing people for change (2nd ed.). New York: Guilford Press. 
Miller, W. R., Yahne, C. E., \& Tonigan, J. S. (2003). Motivational interviewing in drug abuse services: A randomized trial. Journal of Consulting and Clinical Psychology, 71, 754763. doi:10.1037/0022-006X.71.4.754

Nixon, R. D. V., Sweeney, L., Erickson, D. B., \& Touyz, S. W. (2003). Parent-Child Interaction Therapy: A comparison of standard and abbreviated treatments for oppositional defiant preschoolers. Journal of Consulting and Clinical Psychology, 71, 251-260. doi:10.1037/0022-006X.71.2.251

Nix, R. L., Bierman, K. L., \& McMahon, R. J. (2009). How attendance and quality of participation affect treatment response to parent management training. Journal of Consulting and Clinical Psychology, 77, 429-438. doi:10.1037/a0015028

Nock, M. K. \& Ferriter, C. (2005). Parent management of attendance and adherence in child and adolescent therapy: A conceptual and empirical review. Clinical Child and Family Psychological Review, 8, 149-165. doi:10.1007/s10567-005-4753-0

Ogden, T., Hagen, K, A. (2008). Treatment effectiveness of parent management training in Norway: A randomized controlled trial of children with conduct problems. Journal of Consulting and Clinical Psychology, 4, 607-621. doi:10.1037/0022-006X.76.4.607

Sanders, M. R., Kirby, J. N., Tellegen, C. L., \& Day, J. J. (2014). The Triple P-Positive Parenting Program: A systematic review and meta-analysis of a multi-level system of parenting support. Clinical Psychology Review, 34, 337-357.

Schulz K.F., Altman, D. G., \& Moher D. for the CONSORT Group. (2010). CONSORT 2010 Statement: Updated guidelines for reporting parallel group randomised trials. Annals of Internal Medicine, 152, 726-732.

Thomas, R., Abell, B., Webb, H. J., Avdagic, E., \& Zimmer-Gembeck, M. J. (2016). The effectiveness of parent-child interaction therapy on child externalising behaviour: A systematic review and meta-analysis. Manuscript submitted for publication. 
Thomas, R., Zimmer-Gembeck, M. J. (2007). Behavioral outcomes of Parent-Child Interaction Therapy and Triple $\mathrm{P}-$ Positive Parenting Program: A review and metaanalysis. Journal of Abnormal Child Psychology, 35, 475-495. doi:10.1007/s10802007-9104-9

Thomas, R., \& Zimmer-Gembeck, M. J. (2011). Accumulating evidence for best practice: The case of Parent-Child Interaction Therapy and child maltreatment. Child Development, 82, 177-192.

Thomas, R., Zimmer-Gembeck, M. J. (2012). Parent-Child Interaction Therapy: An evidence-based treatment for child maltreatment. Child Maltreatment, 18, 108-121. doi:10.1177/1077559512459555

Timmer, S. G., Ho, L. K. L., Urquiza, A. J., Zebell, N. M., Garcia, E. F., \& Boys, D. (2011). The effectiveness of Parent-Child Interaction Therapy with depressive mothers: The changing relationship as the agent of individual change. Child Psychiatry and Human Development, 42, 406-423. doi:10.1007/s10578-011-0226-5

Vasilaki, E., Hosier, S., \& Cox, W. (2006). The efficacy of motivational interviewing as a brief intervention for excessive drinking: A meta-analytic review. Alcohol and Alcoholism, 41, 328-335. doi:10.1093/alcalc/agl

Waters, A., Farrell, L., Zimmer-Gembeck, M., Milliner, E., Tiralongo, E., Donovan, C., .. . Ollendick, T. (2014). Augmenting one-session treatment of children's specific phobias with attention training to positive stimuli. Behaviour Research and Therapy, 62, 107119. doi:10.1016/j.brat.2014.07.020

Webster-Stratton, C., \& Reid, J. M. (2010) The Incredible Years parents, teachers, and children training series: A multifaceted treatment approach for young children with conduct disorders. In JR Weisz, AE Kazdin (Eds.). Evidence-based psychotherapies for children and adolescents. (pp. 194-210). New York: Guilford Press. 
Table 1

Treatment Effects, Means and Standard Deviations

\begin{tabular}{|c|c|c|c|c|c|c|c|c|c|}
\hline \multirow[b]{2}{*}{ Measures } & \multirow[b]{2}{*}{ Group } & \multicolumn{2}{|c|}{ Pre } & \multicolumn{2}{|c|}{ Post } & \multirow{2}{*}{$\begin{array}{l}\text { 95\% CI of } \\
\text { difference }\end{array}$} & \multirow{2}{*}{$\begin{array}{c}\text { Group } x \\
\text { time } \\
F\end{array}$} & \multirow[b]{2}{*}{$p$} & \multirow{2}{*}{$\begin{array}{c}\text { Effect Size } \\
\qquad d\end{array}$} \\
\hline & & $M$ & $S D$ & $M$ & $S D$ & & & & \\
\hline \multirow{2}{*}{ Externalizing behaviors* } & M/PCIT & 64.69 & 8.72 & 56.84 & 9.13 & $5.46,10.22$ & 5.70 & .004 & 0.09 \\
\hline & Waitlist & 60.99 & 10.16 & 58.08 & 12.39 & $0.39,5.44$ & & & \\
\hline \multirow{2}{*}{ Internalizing symptoms* } & M/PCIT & 55.16 & 10.61 & 50.76 & 10.53 & $1.81,7.00$ & 4.04 & .02 & 0.06 \\
\hline & Waitlist & 52.75 & 9.95 & 51.25 & 11.57 & $-1.25,4.25$ & & & \\
\hline \multirow[t]{3}{*}{ Child behavior problems ${ }^{+}$} & S/PCIT & 19.15 & 7.68 & 10.40 & 6.96 & $6.58,10.92$ & & & \\
\hline & M/PCIT & 19.47 & 7.17 & 10.53 & 7.51 & $6.89,10.98$ & 12.97 & $<.001$ & 0.18 \\
\hline & Waitlist & 17.85 & 8.30 & 15.78 & 9.44 & $-0.10,4.25$ & & & \\
\hline \multirow[t]{3}{*}{ Child behavior intensity ${ }^{\dagger}$} & $\mathrm{S} / \mathrm{PCIT}$ & 148.63 & 37.84 & 121.60 & 33.78 & $18.82,35.23$ & & & \\
\hline & M/PCIT & 155.44 & 30.77 & 123.22 & 26.07 & $24.49,39.96$ & 7.53 & .001 & 0.11 \\
\hline & Waitlist & 145.15 & 33.73 & 134.33 & 37.16 & $2.62,19.03$ & & & \\
\hline \multirow[t]{3}{*}{ Stress due to the parent } & S/PCIT & 143.23 & 30.86 & 130.12 & 29.70 & $7.89,18.33$ & & & \\
\hline & M/PCIT & 142.76 & 28.68 & 134.22 & 26.36 & $3.61,13.46$ & 3.62 & .03 & 0.06 \\
\hline & Waitlist & 145.19 & 26.34 & 142.10 & 26.55 & $-2.13,8.31$ & & & \\
\hline \multirow[t]{3}{*}{ Stress due to the child } & S/PCIT & 133.00 & 24.86 & 115.23 & 23.94 & $12.76,22.79$ & & & \\
\hline & M/PCIT & 137.47 & 22.90 & 120.51 & 24.44 & $12.22,21.69$ & 12.83 & $<.001$ & 0.17 \\
\hline & Waitlist & 128.01 & 23.79 & 126.23 & 28.35 & $-3.24,6.80$ & & & \\
\hline
\end{tabular}

Note. $N$ was 45 for M/PCIT, 40 for S/PCIT, and 40 for waitlist. ${ }^{*}$ Child Behavior Checklist, ${ }^{\dagger}$ Eyberg Child Behavior Inventory 
Table 2

Intent to Treat Analyses - Treatment Effects, Means and Standard Deviations

\begin{tabular}{|c|c|c|c|c|c|c|c|c|c|}
\hline \multirow[b]{2}{*}{ Measures } & \multirow[b]{2}{*}{ Group } & \multicolumn{2}{|c|}{ Pre } & \multicolumn{2}{|c|}{ Post } & \multirow[t]{2}{*}{$\begin{array}{l}\text { 95\% CI of } \\
\text { difference }\end{array}$} & \multirow{2}{*}{$\begin{array}{c}\text { Group } \mathrm{x} \\
\text { time } \\
F\end{array}$} & \multirow[b]{2}{*}{$p$} & \multirow{2}{*}{$\begin{array}{c}\text { Effect Size } \\
\qquad d\end{array}$} \\
\hline & & $M$ & $S D$ & $M$ & $S D$ & & & & \\
\hline \multirow[t]{3}{*}{ Externalizing behaviors* } & S/PCIT & 63.53 & 10.52 & 58.00 & 12.73 & $3.67,7.37$ & \multirow{3}{*}{3.19} & \multirow{3}{*}{.04} & \multirow{3}{*}{.03} \\
\hline & $\mathrm{M} / \mathrm{PCIT}$ & 63.97 & 9.03 & 59.39 & 9.71 & $2.93,6.23$ & & & \\
\hline & Waitlist & 61.44 & 9.86 & 59.28 & 11.74 & $0.19,4.13$ & & & \\
\hline \multirow[t]{3}{*}{ Internalizing symptoms* } & S/PCIT & 54.33 & 10.24 & 49.69 & 11.33 & $2.76,6.53$ & \multirow{3}{*}{3.29} & \multirow{3}{*}{.04} & \multirow{3}{*}{.03} \\
\hline & M/PCIT & 54.95 & 10.25 & 52.38 & 10.38 & $0.90,4.25$ & & & \\
\hline & Waitlist & 54.06 & 11.19 & 52.94 & 12.42 & $-0.89,3.11$ & & & \\
\hline \multirow[t]{3}{*}{ Child behavior problems ${ }^{\dagger}$} & S/PCIT & 19.10 & 7.95 & 13.36 & 8.56 & $4.05,7.43$ & \multirow{3}{*}{6.74} & \multirow{3}{*}{.001} & \multirow{3}{*}{.07} \\
\hline & M/PCIT & 18.05 & 8.01 & 12.83 & 8.47 & $3.72,6.73$ & & & \\
\hline & Waitlist & 17.28 & 8.39 & 15.74 & 9.19 & $-0.26,3.33$ & & & \\
\hline \multirow[t]{3}{*}{ Child behavior intensity $^{\dagger}$} & S/PCIT & 148.48 & 38.19 & 130.75 & 37.84 & $11.51,23.94$ & \multirow{3}{*}{3.45} & \multirow{3}{*}{.03} & \multirow{3}{*}{.04} \\
\hline & M/PCIT & 152.14 & 34.03 & 133.31 & 33.66 & $13.30,24.36$ & & & \\
\hline & Waitlist & 145.44 & 34.07 & 137.43 & 36.97 & $1.41,14.63$ & & & \\
\hline \multirow[t]{3}{*}{ Stress due to the parent } & S/PCIT & 147.34 & 29.37 & 138.75 & 30.47 & $1.81,8.17$ & \multirow{3}{*}{2.90} & \multirow{3}{*}{.05} & \multirow{3}{*}{.03} \\
\hline & $\mathrm{M} / \mathrm{PCIT}$ & 140.27 & 28.54 & 135.29 & 27.09 & $5.03,12.17$ & & & \\
\hline & Waitlist & 142.01 & 25.95 & 139.72 & 25.86 & $-1.51,6.09$ & & & \\
\hline \multirow[t]{3}{*}{ Stress due to the child } & S/PCIT & 134.59 & 25.34 & 122.93 & 26.89 & $6.59,13.23$ & \multirow{3}{*}{8.05} & \multirow{3}{*}{$<.001$} & \multirow{3}{*}{.08} \\
\hline & M/PCIT & 132.53 & 23.74 & 122.62 & 24.03 & $7.93,15.38$ & & & \\
\hline & Waitlist & 130.23 & 25.71 & 128.91 & 29.03 & $-2.63,5.28$ & & & \\
\hline
\end{tabular}

Note. $N$ was 77 for M/PCIT, 61 for S/PCIT, and 54 for waitlist. *Child Behavior Checklist, ${ }^{\dagger}$ Eyberg Child Behavior Inventory 


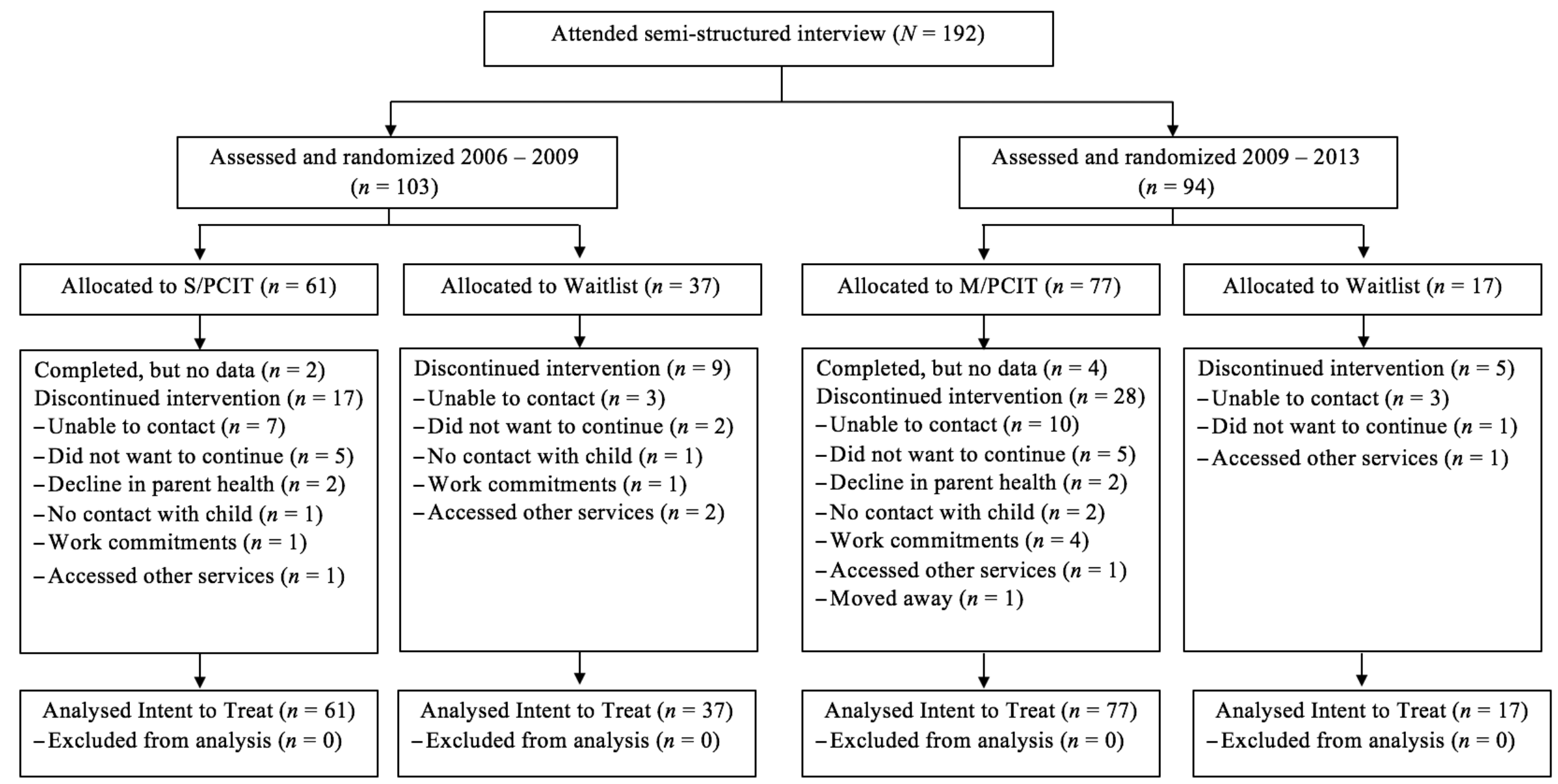

Figure 1. Consort flow diagram of participants through the study. 


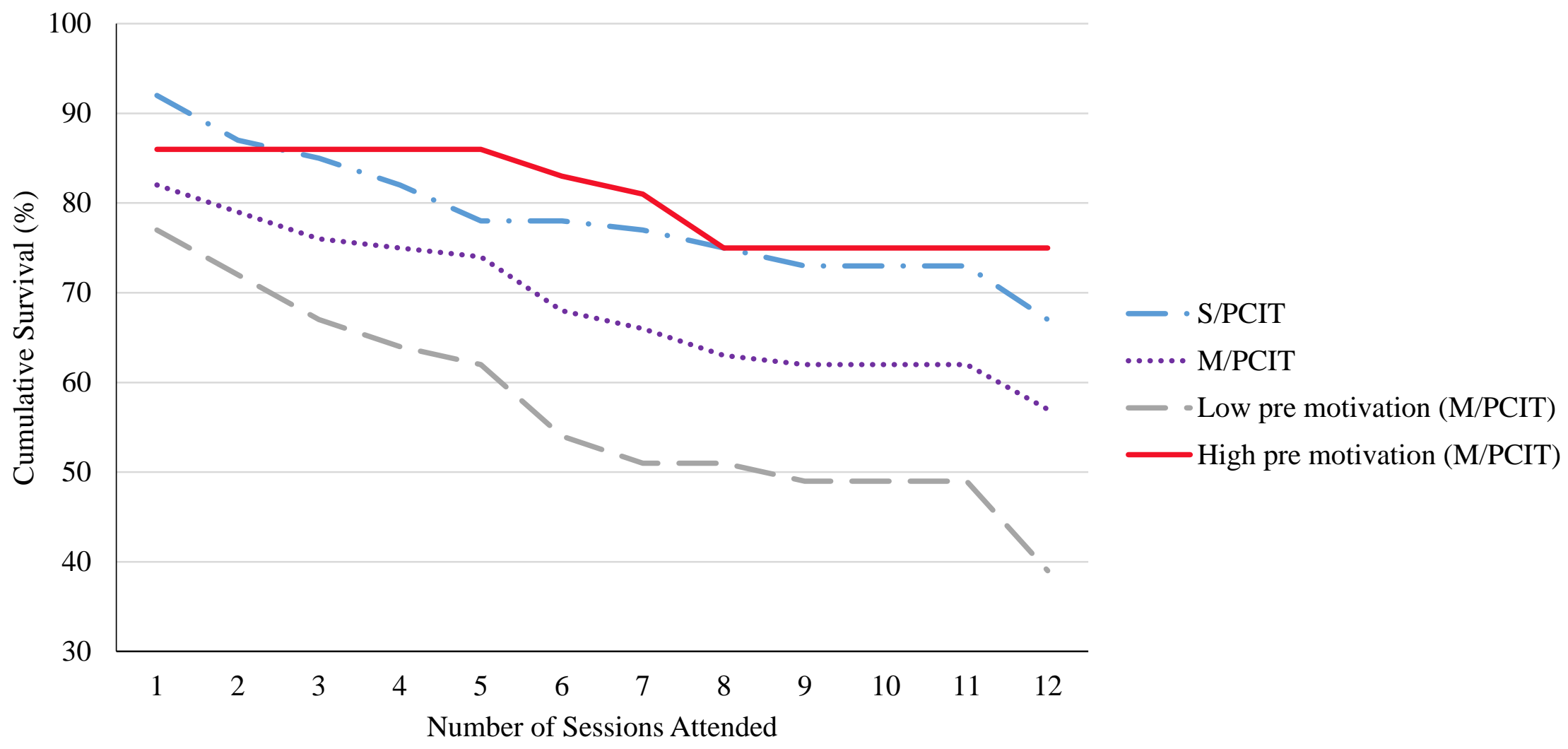

Figure 2. Survival plots according to active treatment group and level of motivation in the M/PCIT group at pre-assessment.

S/PCIT $N=61, \mathrm{M} / \mathrm{PCIT} N=77$, Low pre motivation $N=39$, High pre motivation $N=36$ (two participants were missing pre-assessment of motivation and were excluded from this subgroup analysis). 